\title{
An artificial flexible robot arm based on pneumatic muscle actuators
}

\author{
Jyh-Chyang Renn* and Bo-Han Li \\ Department of Mechanical Engineering, National Yunlin University of Science and Technology, No. 123, University Road Sec. 3, \\ Douliou, 640 Yunlin, Taiwan, R.O.C.
}

\begin{abstract}
The purpose of this paper is to develop a novel human-friendly artificial flexible robot arm using four parallel-connected pneumatic muscle actuators (PMAs). The PMA is a flexible silicone rubber actuator which has some behaviors nearest to the real biological muscle including translational and rotational motions. An inverse kinematic model for the motion control is also developed. Finally, from experiment results, it is proved that not only the axial contraction control of a single PMA but also the attitude control of the whole pneumatic flexible robot arm using PID controller are satisfactory.
\end{abstract}

\section{Introduction}

The pneumatic muscle actuator (PMA) is a soft, deformable fluid actuator able to contract and to exert a tension force when supplied with a given air pressure. One advantage of the PMA is its ability to simulate the real human muscle behaviors and motions. Therefore, it has been widely discussed and researched in the past several years. On the other hand, due to its soft surface, the PMA can work with human, contact the human body and handle fragile objects. Consequently, such an actuator already finds many applications in the field of medical engineering, medical welfare system. For examples, the upper limb muscle actuator [1], powerassist wear [2], bio-mimetic actuators [3], rehabilitation robot $[4,5]$, biceps and triceps actuator [6], and so on. In this paper, a novel human-friendly artificial flexible robot arm using four parallel-connected PMAs is developed. Thanks to the high power-to-weight ratio of the PMA, it is expected that such an artificial arm can help strengthen the force output of a normal human arm or assist some handicapped person. Regarding the control of the artificial arm, one significant achievement of this study is the utilization of only two pneumatic proportional directional control valves to control all four parallel PMAs simultaneously. Thus, the hardware structure can be simplified while the attitude control of the proposed artificial flexible robot arm is still achieved successfully. In the following, two different structures of PMA are firstly outlined.

\section{Translational and rotational PMA}

Figure 1 shows the basic translational type of PMA. It consists of silicon rubber tube covered with a thin fiberglass layer. When the compressed air is supplied to the PMA, it contracts in the axial direction as shown in Fig. 1. The contraction length reaches $10 \mathrm{~mm}$ corresponding to maximal inlet pressure of 6 bar. Another commonly used PMA is the rotational type as shown in Fig. 2. Compared to scheme shown in Fig. 1, a thin metal sheet is inserted between the silicon rubber tube and fiberglass surface. Since the metal sheet is not deformable, the PMA rotates when the compressed air is supplied as shown in Fig. 2. The rotational angle reaches $30^{0}$ when the inlet pressure is the maximal 6 bar.

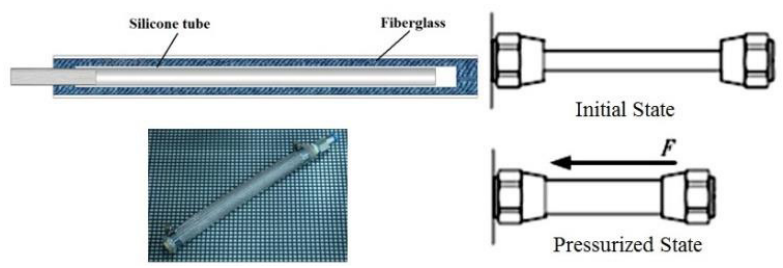

Fig. 1. Translational PMA.

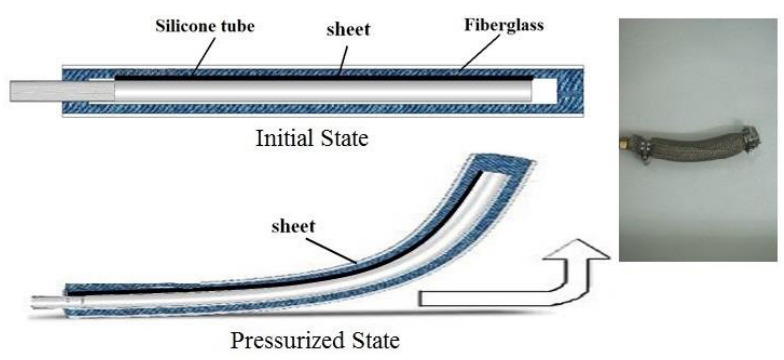

Fig. 2. Rotational PMA.

\section{Structure of artificial flexible robot arm}

As shown in Fig. 3, the design of proposed artificial flexible robot arm is based on closed and parallel linkage mechanism. It consists of two main rigid bodies. One is not movable and hence is called the base while the other is movable and is called the platform. Four parallel translational PMAs serve as the motion constraints and connect the fixed base with the movable platform. In addition, four universal joints are utilized at the platform end. The attitude controls, including circular, pitch and yaw, can be achieved by controlling the deformations of four translational PMAs.

Corresponding author: rennjc@yuntech.edu.tw 


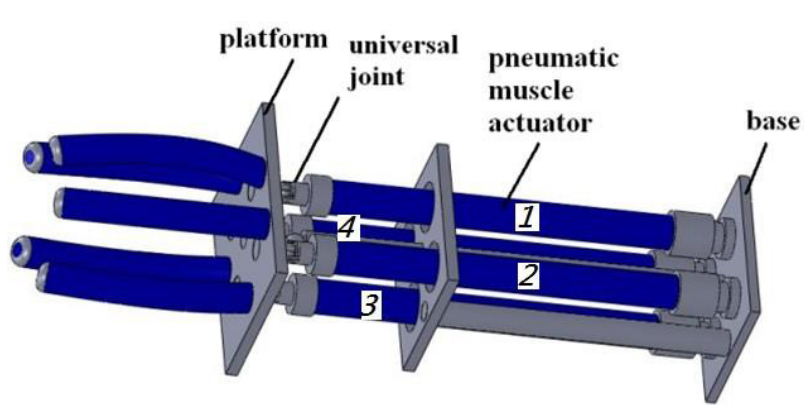

Fig. 3. Scheme of the proposed artificial flexible robot arm.

On the other hand, a soft pneumatic gripper based on four rotational PMAs is also mounted at the other end of the platform to simulate the catch motion of human fingers. Figure 4 shows the real picture of the implemented artificial flexible robot arm mounted on a tripod. Finally, to control the attitude of the movable platform, the contraction lengths of all four PMAs have to be determined by the inverse kinematic model.

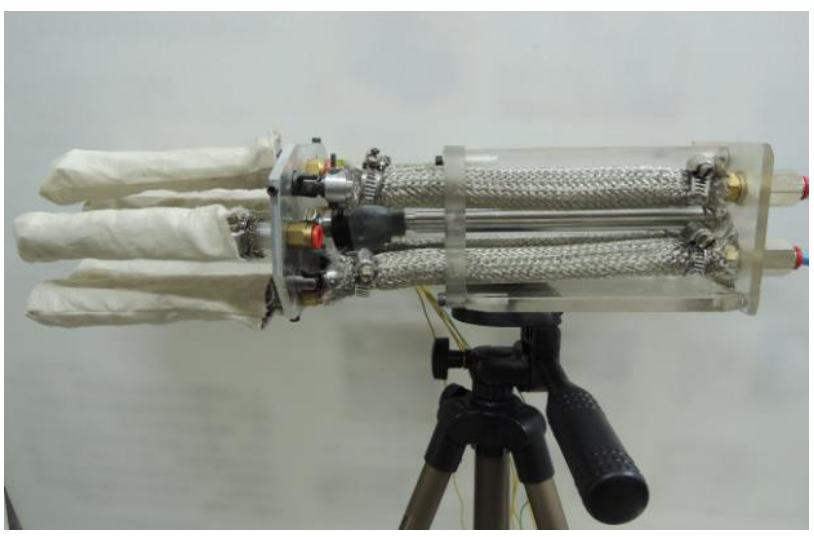

Fig. 4. Real picture of the implemented artificial flexible robot arm.

\section{Inverse kinematic model}

The inverse kinematics that maps Cartesian space into joint space is inevitably necessary for the attitude control [5-7]. Using Cartesian coordinate, the coordinate system $\{\mathrm{B}\}$ for the base and coordinate system $\{\mathrm{P}\}$ for the movable platform can be defined as shown in Fig. 5. Let the matrix ${ }_{P}^{B} \mathbf{R}$ be the attitude orientation matrix of platform coordinate relative to base coordinate and the angles of pitch, roll and yaw of the base coordinate are denoted by $\gamma, \beta$ and $\alpha$. Thus, three orientation matrices for the base coordinate can be derived.

$$
\begin{aligned}
& \mathbf{R}\left(X_{B}, \gamma\right)=\left[\begin{array}{ccc}
1 & 0 & 0 \\
0 & c \gamma & -s \gamma \\
0 & s \gamma & c \gamma
\end{array}\right], \mathbf{R}\left(Y_{B}, \beta\right)=\left[\begin{array}{ccc}
c \beta & 0 & s \beta \\
0 & 1 & 0 \\
-s \beta & 0 & c \beta
\end{array}\right] \\
& \text { and } \mathbf{R}\left(Z_{B}, \alpha\right)=\left[\begin{array}{ccc}
c \alpha & -s \alpha & 0 \\
s \alpha & c \alpha & 0 \\
0 & 0 & 1
\end{array}\right] .
\end{aligned}
$$

where $c \alpha$ denotes $\cos (\alpha)$, s $\alpha$ denotes $\sin (\alpha)$, and so on. Furthermore, the attitude orientation matrix ${ }_{P}^{B} \mathbf{R}$ can be calculated by

$$
\begin{aligned}
{ }_{P}^{B} \mathbf{R} & =\mathbf{R}_{Z_{B}, \alpha} \cdot \mathbf{R}_{Y_{B}, \beta} \cdot \mathbf{R}_{X_{B}, \gamma} \\
& =\left[\begin{array}{ccc}
c \alpha & -s \alpha & 0 \\
s \alpha & c \alpha & 0 \\
0 & 0 & 1
\end{array}\right]\left[\begin{array}{ccc}
c \beta & 0 & s \beta \\
0 & 1 & 0 \\
-s \beta & 0 & c \beta
\end{array}\right]\left[\begin{array}{ccc}
1 & 0 & 0 \\
0 & c \gamma & -s \gamma \\
0 & s \gamma & c \gamma
\end{array}\right] \\
& =\left[\begin{array}{ccc}
c \alpha \cdot c \beta & c \alpha \cdot s \beta \cdot s \gamma-s \alpha \cdot c \gamma & c \alpha \cdot s \beta \cdot c \gamma+s \alpha \cdot s \gamma \\
s \alpha \cdot c \beta & s \alpha \cdot s \beta \cdot s \gamma+c \alpha \cdot c \gamma & s \alpha \cdot s \beta \cdot c \gamma-c \alpha \cdot s \gamma \\
-s \beta & c \beta \cdot s \gamma & c \beta \cdot c \gamma
\end{array}\right] \\
& =\left[\begin{array}{lll}
r_{11} & r_{12} & r_{13} \\
r_{21} & r_{22} & r_{23} \\
r_{31} & r_{32} & r_{33}
\end{array}\right] .
\end{aligned}
$$

From the vector diagram shown in Fig. 6, the vector ${ }^{B} \boldsymbol{d}=\left[\begin{array}{lll}x & y & z\end{array}\right]^{T}$ represents the vector of platform center relative to the base coordinate. After some derivations, the required length vector ${ }^{B} \boldsymbol{q}_{i}=\left[\begin{array}{lll}q_{i x} & q_{i y} & q_{i z}\end{array}\right]^{T}$ for every PMA at pressurized states can be obtained as follows.

$$
{ }^{\mathrm{B}} \mathbf{q}_{i}={ }^{\mathrm{B}} \mathbf{x}_{i}+{ }^{\mathrm{B}} \mathbf{p}_{i} \text {. }
$$

where

$$
\begin{gathered}
{ }^{\mathrm{B}} \mathbf{x}_{i}={ }^{\mathrm{B}} \mathbf{d}-{ }^{\mathrm{B}} \mathbf{b}_{i} \\
=\left[\begin{array}{c}
x-b_{i x} \\
y-b_{i y} \\
z-b_{i z}
\end{array}\right]=\left[\begin{array}{c}
x-b_{i x} \\
y-b_{i y} \\
z
\end{array}\right]=\left[\begin{array}{c}
\bar{x}_{i} \\
\bar{y}_{i} \\
\bar{z}_{i}
\end{array}\right],
\end{gathered}
$$

and

$$
\begin{gathered}
{ }^{\mathrm{B}} \mathbf{p}_{i}={ }_{P}^{B} \mathbf{R}^{\mathrm{P}} \mathbf{p}_{i} \\
=\left[\begin{array}{lll}
r_{11} & r_{12} & r_{13} \\
r_{21} & r_{22} & r_{23} \\
r_{31} & r_{32} & r_{33}
\end{array}\right]\left[\begin{array}{l}
p_{i x} \\
p_{i y} \\
p_{i z}
\end{array}\right]=\left[\begin{array}{l}
r_{11} p_{i x}+r_{12} p_{i y} \\
r_{21} p_{i x}+r_{22} p_{i y} \\
r_{31} p_{i x}+r_{32} p_{i y}
\end{array}\right]=\left[\begin{array}{c}
u_{i} \\
v_{i} \\
w_{i}
\end{array}\right] .
\end{gathered}
$$

Finally, the length $S_{i}$ of $i^{\text {th }}$ PMA is actually the scalar length of the vector ${ }^{\mathrm{B}} \mathbf{q}_{i}$, that is

$$
S_{i}=\sqrt{\left(\bar{x}_{i}+u_{i}\right)^{2}+\left(\bar{y}_{i}+v_{i}\right)^{2}+\left(\bar{z}_{i}+w_{i}\right)^{2}} \quad(i=1,2,3,4)(6)
$$

This is exactly the closed-form solution of the proposed artificial flexible robot arm. 


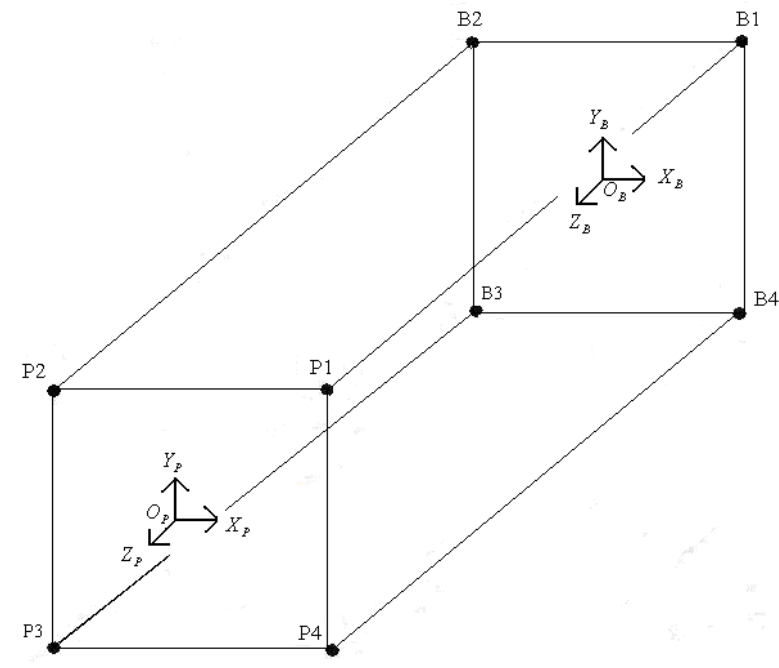

Fig. 5. Coordinate $\{\mathrm{B}\}$ for the base and coordinate $\{\mathrm{P}\}$ for the platform.

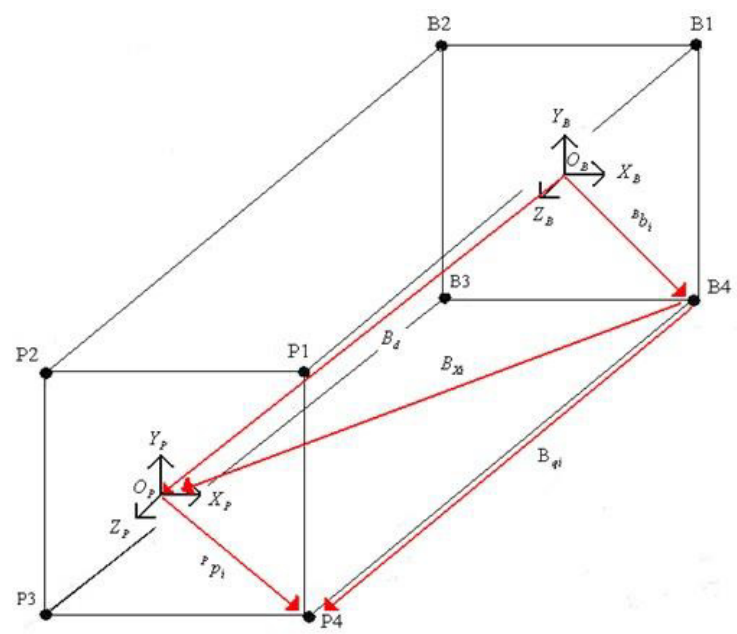

Fig. 6. Vector diagram between two working spaces.

\section{Attitude control of the proposed artificial flexible robot arm}

The pneumatic circuit diagram as well as the control scheme for the proposed artificial flexible robot arm is shown in Fig. 7. In addition to above-mentioned robot arm, one directional $5 / 2$ valve and two proportional directional valves (FESTO MPYE-5-1/8LF-010-B) are utilized and serve as the main control elements. The $5 / 2$ directional valve controls the motion of soft gripper. When the pressurized air is supplied to the soft gripper, four pneumatic muscle fingers rotate simultaneously and form the catch motion. On the other hand, if the pressurized air in the pneumatic muscle fingers is exhausted to atmosphere, four PMAs will return to their initial states. Thus, the release motion can also be implemented.

Instead of four proportional pressure control valves, only two proportional directional control valves are required to control all four parallel-connected translational PMAs. This is chiefly because one proportional directional control valve provides two outlet ports, denoted by A and B. In addition, these two outlet ports are further connected to two PMAs respectively. Thus, one proportional directional control valve is adequate for the control of two PMAs. As an example, for the pitch control, the upper pair of PMA (1) and (2) as well as the lower pair of PMA (3) and (4) shown in Fig. 3 are sequentially activated. On the other hand, one proportional pressure control valve has only one outlet port. Therefore, four proportional pressure control valves are inevitable for the control of all four PMAs. Disadvantages of such a pressure control scheme, however, are bulky, quite complicated and more expensive. Another important sensor utilized in this study is a gyro (Free-scale MMA7361) that detects and feedbacks the real attitude of the movable platform. The mounting location of this gyro is exactly at the center position of the platform which meets the derivation of forward and inverse kinematic equations. Finally, the closed-loop control block diagram using PID controller is shown in Fig. 8.

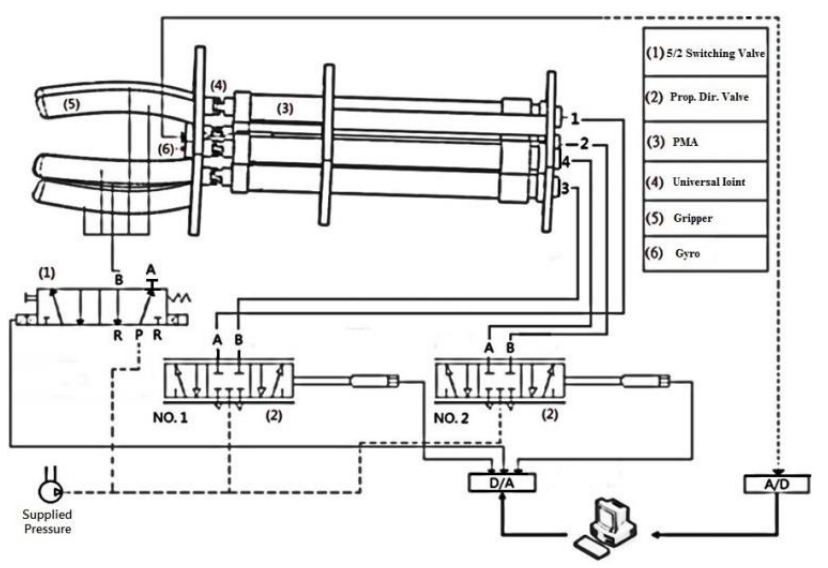

Fig. 7. Pneumatic circuit diagram as well as the control scheme.

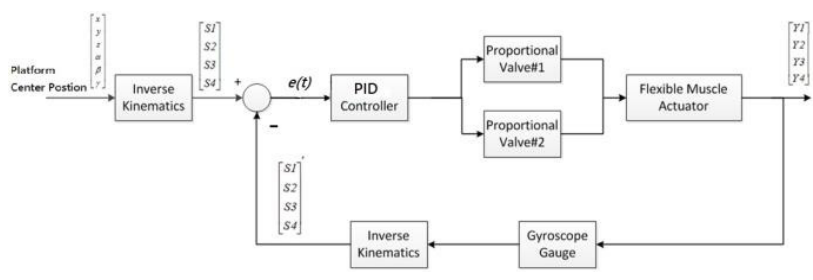

Fig. 8. Closed-loop control block diagram using PID controller.

\section{Experimental results of circular tests}

Before conducting the circular test, the axial contraction control of one single PMA using PID controller is firstly executed. The initial length of this single PMA is set to be $200 \mathrm{~mm}$. Moreover, five different contraction lengths of PMA from $2 \mathrm{~mm}$ to $10 \mathrm{~mm}$ are tested. From the experimental results shown in Fig. 9, it is observed that the response is very slow and the position control behavior is quite nonlinear. However, the steady-state 
error is only around $0.2 \mathrm{~mm}$ and is satisfactory. After further frequency response analysis, the bandwidth of the developed PMA is estimated to be $0.24 \mathrm{~Hz}$. For the circular test, the path of the platform's center position using Cartesian coordinate can be defined as

$X=r \cos (\theta)=r \cos (2 \pi f)$,

$Y=r \sin (\theta)=r \sin (2 \pi f)$.

Three different radii are chosen, that is $r=12 \mathrm{~mm}, r=$ $9 \mathrm{~mm}$ and $r=6 \mathrm{~mm}$. The initial lengths for all four PMAs are also set to be $Z=200 \mathrm{~mm}$ and the frequency of circular test is set to be $f=0.01 \mathrm{~Hz}$. The corresponding results using PID controller are shown in Fig. 10. The maximal error is found to be $0.3 \mathrm{~mm}$ which also implies the good and acceptable circular test precision of the proposed artificial flexible robot arm based on PMA.

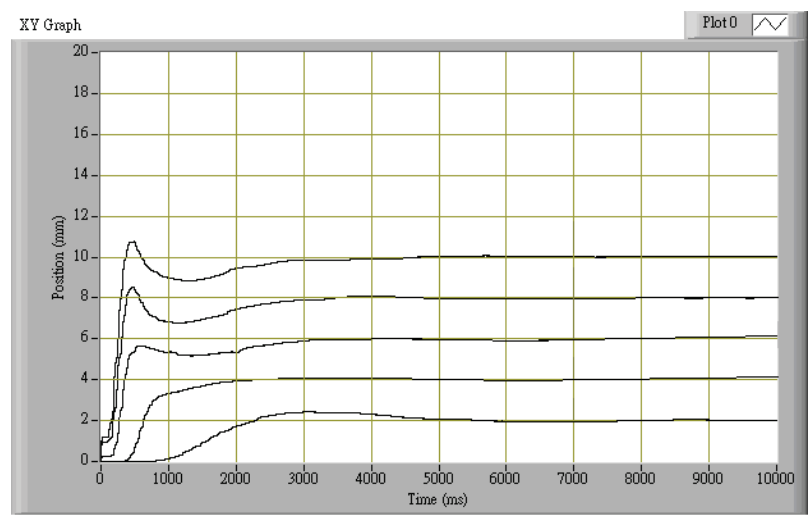

Fig. 9. Five different length contractions control results using PID controller.

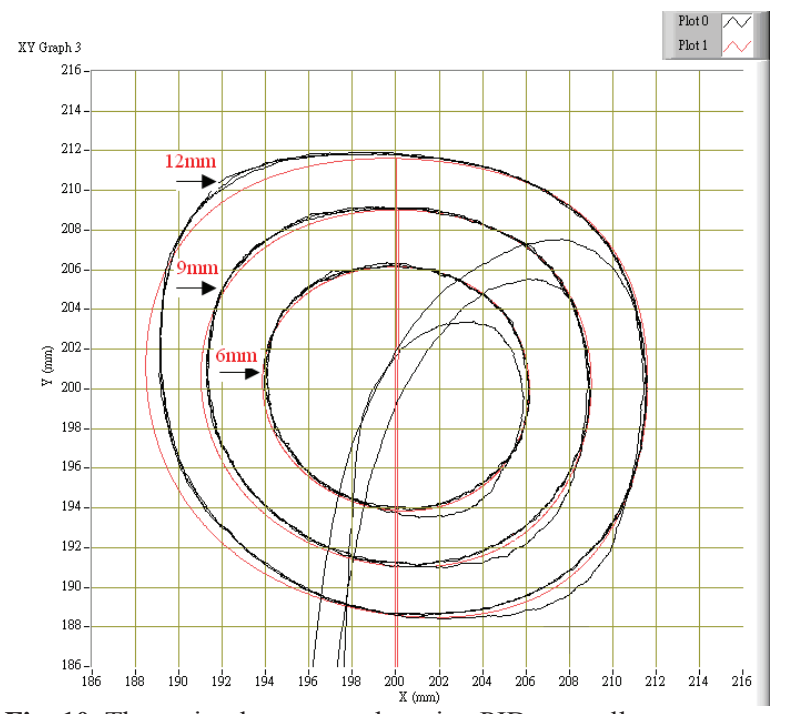

Fig. 10. Three circular test results using PID controller.
Table 1. Specifications of the artificial flexible robot arm.

\begin{tabular}{|ll|c|}
\hline \multicolumn{1}{|c|}{ Items } & Spec. \\
\hline 1. & Initial length of PMA & $200 \mathrm{~mm}$ \\
\hline 2. & Max. contraction length of PMA & $10 \mathrm{~mm}$ \\
\hline 3. & Max. pitch angle & $\pm 15^{\circ}$ \\
\hline 4. & Max. yaw angle & $\pm 15^{\circ}$ \\
\hline 5. & Supplied pressure & $5 \mathrm{bar}$ \\
\hline 6. & Bandwidth (one PMA) & $0.24 \mathrm{~Hz}$ \\
\hline
\end{tabular}

\section{Conclusions}

In this paper, a novel human-friendly artificial flexible robot arm using four parallel PMAs is successfully developed and realized. Three different motions, including pitch, yaw and circular, can be implemented. Table 1 summarizes some specifications of the proposed artificial flexible robot arm. To get the maximal angle specifications, maximal input $(10 \mathrm{~V})$ to the proportional valve is used to control the attitude of robot. On the other hand, to find the bandwidth, the same maximal amplitude input to the proportional valve, but with various frequencies, is employed to control the contraction of PMA. The $-3 \mathrm{~dB}$ frequency of the frequency response is denoted as the bandwidth. In the future, the PMA and the artificial flexible robot arm may find more potential applications in the field of medical engineering or medical welfare system, etc.

\section{Acknowledgment}

The financial support of the Ministry of Science and Technology under grant number MOST- 104- 2221- E- 224017 is greatly appreciated.

\section{References}

1. M. Aragane, "Power assist wear for upper limb driven by sheet-like pneumatic rubber muscle," Proceedings of $7^{\text {th }}$ JFPS International Symposium on Fluid Power, Toyama, Japan, pp. 787-792, 2008

2. T. Noritsugu, D. Sasaki and M. Takaiwa, "Application of pneumatic soft mechanism to a human friendly robot," Proceedings of $5^{\text {th }}$ JFPS International Symposium on Fluid Power, Nara, Japan, pp. 61-66, 2002

3. D. G. Caldwell, N. Tsagarakis, and G. A. MedranoCerdas, 'Bio-mimetic actuators: polymeric pseudo muscle actuators and pneumatic muscle actuators for biological emulation," Mechatronics, 10, pp. 499-530, 2000

4. S. D. Prior and A. S. White, "Measurement and simulation of a pneumatic muscle actuator for a rehabilitation robot," Simulation Practice and Theory, pp. 81-117, 1995 
5. T. Noritsugu and T. Tanaka, "Application of rubber artificial muscle manipulator as a rehabilitation robot," IEEE/ ASME Trans. Mechatronics, 2, pp. 259-267, 1997

6. J. H. Lilly, "Adaptive Tracking for pneumatic muscle actuators in biceps and triceps configurations," IEEE Transactions on Neural System and Rehabilitation Engineering, 11 (3), pp. 333-339, 2003 